\title{
Model of Hydrophobic Attraction in Two and Three Dimensions
}

\author{
G.T. Barkema and B. Widom \\ Instituut voor Theoretische Fysica, Universiteit Utrecht, \\ Princetonplein 5, 3584 CC Utrecht, The Netherlands
}

(March 22, 2000)

\begin{abstract}
An earlier one-dimensional lattice model of hydrophobic attraction is extended to two and three dimensions and studied by Monte Carlo simulation. The solvent-mediated contribution to the potential of mean force between hydrophobic solute molecules and the solubility of the solute are determined. As in the earlier model, an inverse relation is observed between the strength and range of the hydrophobic attraction. The mean force no longer varies monotonically with distance, as it does in one dimension, but has some oscillations, reflecting the greater geometrical complexity of the lattice in the higher dimensions. In addition to the strong attraction at short distances, there is now also a local minimum in the potential of depth about $k T$ at a distance of three lattice spacings in two dimensions and one of depth about $2 k T$ at a distance of two lattice spacings in three dimensions. The solubility of the solute is found to decrease with increasing temperature at low temperatures, which is another signature of the hydrophobic effect and also agrees with what had been found in the one-dimensional model.
\end{abstract}

\section{INTRODUCTION}

A one-dimensional lattice madel of hydrophobic attraction has recently been studiedl. The model incorporates what is believed to be the basic mechanism of the hydrophobic effect; viz., that the accommodation of the solute in the solvent is energetically favorable but sufficiently unfavorable entropically as to result in an increase in free energy. This increase is smaller when the hydrophobes are close together than when they are widely separated, resulting in a net solvent-mediated attraction between them.

The unfavorable entropy of accommodating the solute is achieved in the model by allowing each solvent molecule to have a large number $q$ of possible orientations but requiring two neighboring solvent molecules to be both in a special one of those $q$ states if they are to accommodate a hydrophobic solute in the interstitial site between them. That circumstance in which two neighboring solvent molecules are both in that special orientation is taken to be energetically favorable by an amount previously calledl $u-w$. The condition of hydrophobicity at temperature $T$ was that $k T \ln (q-1)>u-w$, with $k$ Boltzmann's constant. To model the hydrophobic effect realistically, $(u-w) / k$ was taken to be $3000 \mathrm{~K}$ and $q$ was taken to be 110,000 .

It was then a property of the model that forcing the solvent to accommodate a hydrophobic solute greatly restricted (to one state out of $q$ ) the possible orientations of the solvent molecules neighboring the accommodated solute. Such restriction of solvent orientation by the solute is consistent with experimental measurements of NMR relaxation times, which show that the reorientation times of water molecules that neighbor a solute with alkyl groups are about twice as long as in pure water, and that there is an increased activation energy for the reorientation in the presence of the solute over that in pure water.

It was a striking feature of the one-dimensional model that the range and strength of the solvent-mediated attraction between solutes were inversely related to each other; at low temperatures the attraction was weak but long-ranged and at high temperatures it was strong but short-ranged. One of the motivations of the present work was to see if that inverse relation, which is of potential importance in the interpretation of experiment, persists in higher dimensions, and it is indeed found to be so.

It was also found earlier in the one-dimensional model that the solubility of the hydrophobe decreases with increasing temperature, which is consistent with the increasing hydrophobicity that manifests itself in the increasing strength of the hydrophobic attraction. This temperature dependence of the solubility, too, is found here to persist in the two- and three-dimensional models. It is also found, as in the one-dimensional model, that if the energy of interaction of the hydrophobic solute with its solvent neighbors (earlier called $v$ ) is greater than the magnitude $u-w>0$ of the favorable energy of solvent alignment, then the solubility, after first decreasing with increasing temperature, reaches a minimum and then increases.

The two- and three-dimensional versions of the model are defined in the following section. Also in Section III are the basic formulas that relate the solvent-mediated part of the potential of mean force and the solubility to the quantities that are measured in the simulations. The details of the simulations are in Section III, where the results are also displayed. These are discussed and summarized in the concluding Section IV.

\section{THE TWO- AND THREE-DIMENSIONAL MODELS}

The two-dimensional model is pictured in Fig. 1. At each site of a square lattice there is a solvent molecule that may be in any of a large number, $q$, of different states (orientations). Solute molecules may only be accommo- 
dated at the interstitial sites between neighboring pairs of solvent molecules, but only if that site is not already occupied and then only if the two solvent molecules in question are both in a special one of the $q$ states, called state 1 in what follows. The figure shows three solute molecules so accommodated.

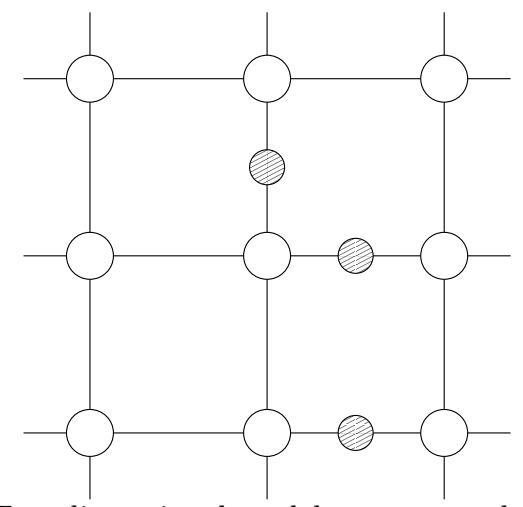

FIG. 1. Two-dimensional model on a square lattice. There is a solvent molecule (open circle) at each lattice site; solute molecules (shaded circles) may only occupy interstitial sites.

The three-dimensional model is defined similarly, and also on a lattice of coordination number 4 , as in two dimensions. This three-dimensional lattice is pictured in Fig. 2. It is obtained by systematically removing two bonds from each vertex of a simple cubic lattice, in such a way that from each site $(i, j, k)$ there remains a bond to the neighboring sites at $(i+1, j, k)$ and $(i-1, j, k)$, as well as to those at $(i, j-1, k)$ and $(i, j, k-1)$ if $i+$ $j+k$ is even but to those at $(i, j+1, k)$ and $(i, j, k+1)$ instead if $i+j+k$ is odd. The lattice is topologically (not metrically) equivalent to the tetrahedral diamond lattice, which is also the lattice of oxygen atoms in the metastable phase of ice called ice $\mathrm{I}_{c}$.

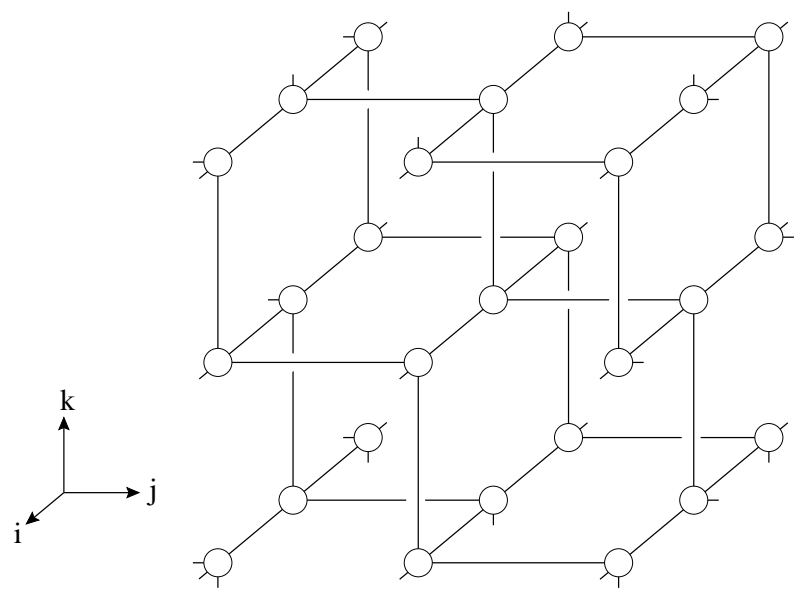

FIG. 2. Four-coordinate lattice in three dimensions obtained by systematically removing two bonds from each vertex of a simple cubic lattice. Circles mark the lattice sites, of which 27 are shown, in three horizontal $(i, j)$ or vertical $(j, k$ or $k, i)$ planes of nine sites each.
In both the two- and three-dimensional versions of the model only neighboring solvent molecules interact with each other, which they do with energy $w$ if both are in the special state 1 and with energy $u>w$ otherwise. The only solvent molecules with which a solute molecule interacts are its immediate neighbors; the sum of its energies of interaction with them is $v$.

Solute molecules may interact with each other but that is irrelevant here; the solute-solute interaction does not enter the expression for the solvent-mediated part of the potential of mean force petween pairs of solutes in the infinitely dilute solution 1 , nor is it relevant for the solubility of the solute, which is so slight that solute-solute interactions are negligible. The solute-solvent interaction parameter $v$ is also absent from the expression for the potential of mean force in the infinitely dilute solution but it is a significant parameter in the solubility.

Because the coordination number of the lattices considered here is 4 , rather than 2 as in one dimension, the values of the parameters $u-w$ and $q$ required to model the hydrophobic effect conveniently but still realistically over the temperature range $275-375 \mathrm{~K}$ are both much smaller than in one dimension. Here, in both the two- and three-dimensional models, they are taken to be $(u-w) / k=1000 \mathrm{~K}$ instead of $3000 \mathrm{~K}$ and $q=1100$ instead of 110,000 . It is still the case, as in the onedimensional model, that $k T \ln (q-1)>u-w$ over the relevant temperature range, and that the extent to which $k T \ln (q-1)$ exceeds $u-w$ increases with increasing temperature — which are the respective conditions required for the hydrophobic effect to manifest itself and to do so with increasing strength as the temperature increases.

Let $p_{11}$ be the probability that in the pure solvent both molecules of a given neighboring pair will be found in the special state 1 ; and let $p(r)$ be the probability that the molecules at two such pairs of sites will all be found to be in the special state 1 when the corresponding two interstitial sites, one associated with each pair, are separated by the metrical distance $r$ (measured in units of the lattice spacing). In the two-dimensional lattice of Fig. 1 the values of $r$ that occur are $r=\sqrt{2} / 2=0.71 ; 1 ; \sqrt{2}=$ $1.41 ; \sqrt{5 / 2}=1.58$; etc. In the three-dimensional lattice of Fig. 2 the values of $r$ that occur are those and also $r=\sqrt{3 / 2}=1.22$, etc.

Let $W(r)$ be the solvent-mediated part of the potential of mean force between two solute molecules a distance $r$ apart, in the limit of infinite dilution. This is the potential of mean force from which the direct interaction between the two solute molecules themselves has been subtracted, and is the part of the mean-force potential that is of interest here. Then, as in the one-dimensional model, 1 W $W(r)$ is given in terms of $p_{11}$ and $p(r)$ by

$$
W(r)=-k T \ln \left[p(r) / p_{11}^{2}\right] .
$$

The quantities $p_{11}$ and $p(r)$ are what are determined in the simulations and $W(r)$ is then obtained from (1). It is a property of the solvent alone, and so depends only 
on the parameters $u-w$ and $q$ but not on $v$.

The solubility is defined as in the earlier onedimensional model 10 One imagines a hypothetical ideal gas of pure solute, of number density $\rho_{g}$, in osmotic equilibrium with the saturated solution; i.e., having a thermodynamic activity equal to that of the solute in the solution. Then if $\rho_{s}$ is the number density of the solute in the solution, the solubility $\Sigma$ is here defined as the dimensionless ratio

$$
\Sigma=\rho_{s} / \rho_{g}
$$

If the solubility is low so that the saturated solution is very dilute, this $\Sigma$ is the Ostwald absorption coefficient and is given by 1

$$
\Sigma=p_{11} \mathrm{e}^{-v / k T}
$$

Thus, $\Sigma$ is obtained once $p_{11}$ is calculated and a value of the solute-solvent interaction energy $v$ is specified.

\section{SIMULATIONS}

Properties of the two- and three-dimensional models presented in the previous Section are explored by means of Monte Carlo simulations. In these simulations, one solvent molecule is placed on each lattice site. For each molecule, we keep track of whether its state is the special state 1 , or not. Solute molecules are not present in the Monte Carlo simulations, since the aim of the simulations is to numerically estimate the quantities $p_{11}$ and $p(r)$, from which the solvent-mediated part of the potential of mean force can be computed using (11).

The dynamics of the models are not specified, and we are therefore free to choose any dynamics that yield the correct equilibrium properties. In our Monte Carlo simulations, the dynamics consist of two processes: 1) each solvent molecule that is in its special state 1 leaves this special state with a rate of $q-1$ per time unit; 2 ) each solvent molecule that is in a different state enters its special state with a rate of $\alpha^{n}$ per time unit, where $\alpha=\exp ((u-w) / k T)$ and $n$ is the number of nearestneighbor sites that are occupied with a solvent molecule in its special state. An efficient implementation of these dynamics is obtained with a BKL-scheme, after Bortz, Kalos and Lebowitzl.

The simulations are performed on a $500 \times 500$ lattice for the two-dimensional model, and a $50 \times 50 \times 50$ lattice for the three-dimensional model, both with periodic boundary conditions in order to reduce finite-size effects. In the initial configuration, no molecule is in its special state. This configuration is then thermalized for 0.1 time units. After thermalization, $p_{11}$ and $p(r)$ are measured every $10^{-3}$ time unit, up to a total time of $10^{3}$ time units, and averaged. In a simulation of this length and for these system sizes, the two-dimensional and three-dimensional model undergo roughly $5.5 \cdot 10^{8}$ and $2.7 \cdot 10^{8}$ local changes, respectively. This procedure is repeated 10 times for each of the temperatures $T=275,300,325,350$, and $375 \mathrm{~K}$, and for the three-dimensional model also at a temperature of $250 \mathrm{~K}$ at which the system is probably metastable, the stable regime being one where almost all atoms are in their special state.

The values for the quantity $p_{11}$ are presented in Table fi, for the two- and three-dimensional models. This quantity is decreasing with increasing temperature. The quantity $p_{11}$ is one of the two factors determining the solubility as defined in equation (3), the other being $\exp (-v / k T)$. While this latter factor is monotonically increasing with increasing temperature for $v>0$, our data show that $p_{11}$ is monotonically decreasing with increasing temperature. For some choices of $v$, the combination of these two factors leads to a solubility that is initially decreasing with increasing temperature (a signature of the hydrophobic effect), but later increasing.

The solvent-mediated part of the potential of mean force $W(r)$ is plotted as a function of the distance $r$ in Fig. 3 for the two-dimensional model, and in Fig. 4 for the three-dimensional model. In the latter figure, the curve obtained at a temperature of $250 \mathrm{~K}$ is dashed, to indicate its likely metastability. In agreement with the one-dimensional model, the strength of the hydrophobic interaction increases with increasing temperature, while its range decreases at the same time: an inverse relation is observed between the strength and range of the hydrophobic interaction, althoug less prominently in three dimensions than in one or two. Unlike the one-dimensional model, the two- and three-dimensional models show oscillations in the mean force, reflecting the greater geometrical complexity of the lattice in higher dimensions. In that sense, the curves resemble more closely what is seen in models in which the solvent is modeled more realistically 4 . In addition to the strong attraction at short distances, there is now also a local minimum in the potential of depth about $k T$ at a distance of three lattice spacings in two dimensions and one of depth about $2 k T$ at a distance of two lattice spacings in three dimensions.

TABLE I. The function $p_{11}$ as a function of temperature $T$, for the two-dimensional (second column) and three-dimensional model (third column). The numbers between brackets are the statistical uncertainties in the last reported digit, obtained from the standard deviation divided by the square root of the number of independent simulations.

\begin{tabular}{l|l|l}
\hline \hline $\mathrm{T}$ & $p_{11}(2 \mathrm{D})$ & $p_{11}(3 \mathrm{D})$ \\
\hline 250 & - & $6.3324(8) \cdot 10^{-5}$ \\
275 & $4.3715(5) \cdot 10^{-5}$ & $3.9119(8) \cdot 10^{-5}$ \\
300 & $2.8156(3) \cdot 10^{-5}$ & $2.7108(4) \cdot 10^{-5}$ \\
325 & $2.0510(3) \cdot 10^{-5}$ & $2.0177(3) \cdot 10^{-5}$ \\
350 & $1.5913(3) \cdot 10^{-5}$ & $1.5791(4) \cdot 10^{-5}$ \\
375 & $1.2876(2) \cdot 10^{-5}$ & $1.2822(2) \cdot 10^{-5}$ \\
\hline \hline
\end{tabular}




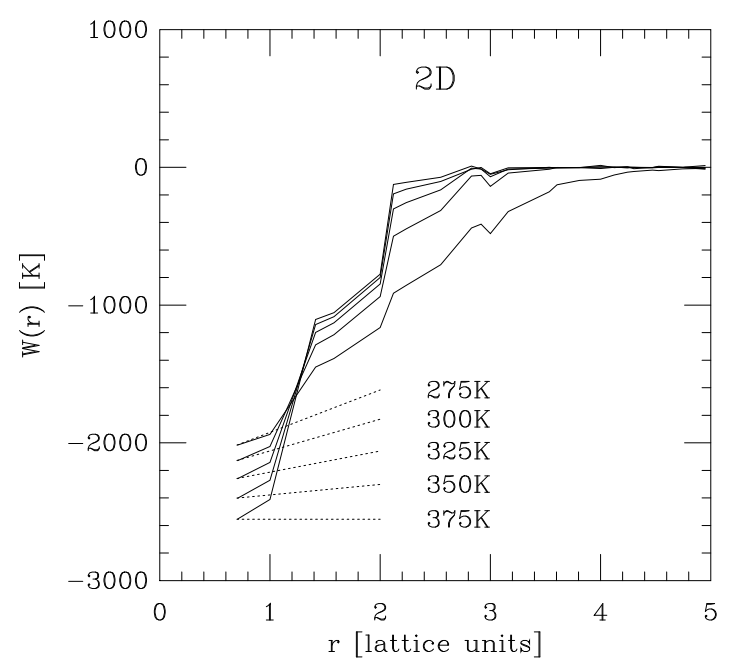

FIG. 3. The solvent-mediated part of the potential of mean force $W(r)$, in degrees Kelvin, as a function of distance $r$ between the solute molecules, in lattice units, for the two-dimensional model. The statistical error in $W(r)$ is at most two Kelvin, too small to be plotted.

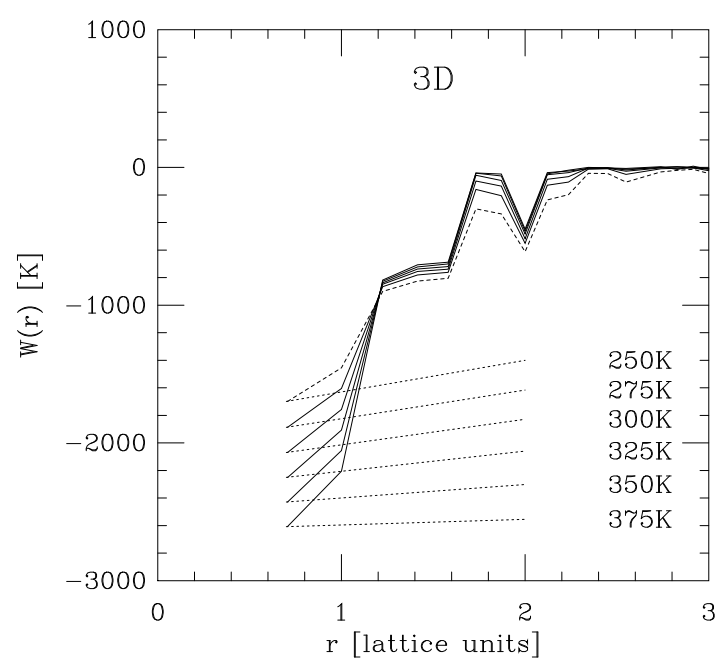

FIG. 4. The solvent-mediated part of the potential of mean force $W(r)$, in degrees Kelvin, as a function of distance $r$ between the solute molecules, in lattice units, for the three-dimensional model. The statistical error in $W(r)$ is at most two Kelvin, too small to be plotted. The curve obtained at a temperature of $250 \mathrm{~K}$ is dashed, to indicate its likely metastability.

\section{SUMMARY AND CONCLUSIONS}

A one-dimensional lattice model of hydrophobic attraction that was studied recently is extended to two and three dimensions. Monte Carlo simulations have been used to determine the solvent-mediated contribution to the potential of mean force between hydrophobic solute molecules, and the solubility of the solute.

As in the one-dimensional model, an inverse relation is observed between the strength and range of the hydrophobic interaction. With increasing temperature, the strength of the hydrophobic interaction increases, while at the same time its range decreases, although the effect is less prominent in three dimensions than in two or one. Unlike in the one-dimensional model, the force no longer varies monotonically with distance, but shows oscillations, reflecting the greater geometrical complexity of the lattice in the higher dimensions.

At low temperatures, the solubility of the solute is found to decrease with increasing temperature. This is another signature of the hydrophobic effect, and also agrees with what had been found in the one-dimensional model.

\section{ACKNOWLEDGEMENTS}

BW is grateful to the Institute of Theoretical Physics and to the Debye Institute of the University of Utrecht for their hospitality in the fall of 1999. His work at Cornell was supported by the U.S. National Science Foundation and the Cornell Center for Materials Research. The authors thank M.D. Zeidler for calling their attention to the work in Reference 2.

\footnotetext{
* Permanent address: Department of Chemistry, Baker Laboratory, Cornell University, Ithaca, New York 14853-1301, USA.

${ }^{1}$ A.B. Kolomeisky and B. Widom, Faraday Discuss. 112 (1999) 81.

${ }^{2}$ H.G. Hertz and M.D. Zeidler, Ber. Bunsengesell. Phys. Chem. 68 (1964) 821.

${ }^{3}$ A.B. Bortz, M.H. Kalos and J.L. Lebowitz, J. Comp. Phys. 17 (1975) 10.

${ }^{4}$ L.R. Pratt and D. Chandler, J. Chem. Phys. 67 (1977) 3683.

${ }^{5}$ M.E. Paulaitis, S. Garde and H.S. Ashbaugh, Curr. Opin. Colloid Interface Sci. 1 (1996) 376.
} 